\title{
Utilization of cobalamin is ubiquitous in early-branching fungal phyla
}

Małgorzata Orłowska, Kamil Steczkiewicz and Anna Muszewska*

Institute of Biochemistry and Biophysics, Polish Academy of Sciences, Pawinskiego 5A, 02-106

Warsaw, Poland

*Corresponding author: Anna Muszewska, musze@ibb.waw.pl

\section{Key words}

Cobalamin, early-diverging fungi, metabolic traits, vitamin B12, fungal evolution

\section{Significance}

Cobalamin dependence is scattered across the tree of life. In particular, fungi and plants have been deemed devoid of cobalamin. We demonstrate that cobalamin is utilized by all phyla of nonDikarya fungal lineages. This observation is supported by the identification of B12-dependent enzymes and cobalamin modifying enzymes in published fungal genomes. We found that cobalamin dependence was a widely distributed trait at least in Opisthokonta, across diverse microbial eukaryotes and likely in the LECA (last eukaryotic common ancestor). Moreover, the genes identified are actively transcribed in many taxa.

\section{Abstract}

Cobalamin is a cofactor present in essential metabolic pathways in animals and one of the watersoluble vitamins. It is a complex compound synthesized solely by prokaryotes. Cobalamin dependence is scattered across the tree of life. In particular, fungi and plants were deemed devoid of cobalamin. We demonstrate that cobalamin is utilized by all non-Dikarya fungi lineages. This observation is supported by the genomic presence of both B12-dependent enzymes and cobalamin modifying enzymes. Fungal cobalamin-dependent enzymes are highly similar to their animal homologs. Phylogenetic analyses support a scenario of vertical inheritance of the cobalamin usage with several losses. Cobalamin usage was probably lost in Mucorinae and at

(C) The Author(s) 2021. Published by Oxford University Press on behalf of the Society for Molecular Biology and Evolution. This is an Open Access article distributed under the terms of the Creative Commons Attribution License (http://creativecommons.org/licenses/by/4.0/), which permits unrestricted reuse, distribution, and reproduction in any medium, provided the original work is properly cited. 
the base of Dikarya which groups most of the model organisms and which hindered B12dependent metabolism discovery in fungi. Our results indicate that cobalamin dependence was a widely distributed trait at least in Opisthokonta, across diverse microbial eukaryotes and was likely present in the LECA.

\section{Introduction}

Cobalamin, also known as vitamin B12, is the most common cobalt-containing compound in nature and one of eight known water-soluble vitamins grouped into B class. Cobalamin is an organometallic complex compound that contains a cobalt atom placed within a corrin ring. Vitamin B12 is derived from uroporphyrinogen III, which is also the first macrocyclic intermediate in a common pathway of heme and chlorophyll biosynthesis (Chatthanawaree 2011); (Dereven'kov et al. 2016); (Frank et al. 2005). In animals, cobalamin is used as a cofactor in myelin formation and thus is crucial for the proper functioning of the nervous system. A deficit of this vitamin in the diet may lead to sensory or motor deficiencies and to degeneration of the spinal cord (Dardiotis et al. 2017).

Biosynthesis of cobalamin takes place only in bacteria and archaea which is quite unique for such a widely used vitamin. It is a very complex process involving more than 30 genes (Roth et al. 1993) collectively conserved only in B12-producing prokaryotes which suggest a common origin of the whole pathway. Nonetheless, animals and some protists, but not fungi, are known to utilize cobalamin in their metabolism so they have to intake this vitamin with food. Interestingly, several eukaryotic microorganisms, including Phytophthora (Oomycota) and Dictyostelium (Amoebozoa), do possess B12-dependent enzymes (Grenville-Briggs et al. 2005); (Crona et al. 2013)). Some algae like Porphyridium purpureum and Amphidinium operculatum, can obtain the cobalamin cofactor from associated bacteria (Croft et al. 2005). Plants and fungi are believed to neither synthesize nor even have a need for the cobalamin (Jah et al. 2002; Duda, Pedziwilk, and Zodrow 1957). Even more, they are regarded as devoid of cobalt at all (Zhang, Ying, and Xu 2019).

In Eukaryotes, B12-dependent enzymes are used in diverse processes ranging from the regeneration of methionine from homocysteine, catabolic breakdown of some amino acids into succinyl-CoA (necessary for citric acid cycle) and proper myelin synthesis. Only seven enzymes from the above pathways seem to be uniquely present in B12-dependent organisms. They either modify cobalamin or use it as a cofactor. The former group contains methylmalonyl Co-A mutaseassociated GTPase Cob (MeaB), cob(I)yrinic acid a,c-diamide adenosyltransferase (CblAdo 
transferase), cyanocobalamin reductase (CbIC), and cobalamin trafficking protein $D(C b I D)$ proteins, while the latter includes methionine synthase $(\mathrm{MetH})$, methylmalonyl-CoA epimerase (MM-CoA epimerase) and methylmalonyl-CoA mutase (MM-CoA mutase) (Kräutler 2012). All these proteins are present in animals, including Holozoa e.g. Monosiga brevicollis. There is one more cobalamin-dependent enzyme that is present uniquely in archaebacteria, eubacteria, and bacteriophages. This enzyme called ribonucleotide reductase class II (RNR class II) takes a part in DNA replication and repair ((Herrick and Sclavi 2007); (Larsson et al. 2004)). For consistency and clarity, we will use the names of human representatives (given above) to tag the above seven enzymes and a bacterial representative for the last one.

RNR is an enzyme that catalyzes the formation of deoxyribonucleotides from ribonucleotides. It plays a pivotal role in the synthesis, reparation, and regulation of the total rate of DNA synthesis (Herrick and Sclavi 2007); (Larsson et al. 2004). RNRs are divided into three classes that are working based on similar mechanisms but using a different compound to generate free radicals. Class I reductases are divided into IA and IB subclasses. These reductases generate tyrosyl free radicals from iron. Subclass IA is distributed in eukaryotes, eubacteria, and viruses. Subclass IB can be found only in eubacteria. Class II reductases use free radicals from cobalamin and are distributed in archaebacteria, eubacteria, and bacteriophages. The same distribution applies to class III, but this class uses a glycine radical (Kolberg 2004). Most eukaryotes, including animals, use class IA reductases, but surprisingly Phytophthora spp. uses cobalamin-dependent class II RNR.

Methionine synthetase (MetH) comes in two variants: cobalamin-dependent MetH (EC 2.1.1.13) and cobalamin-independent MetE (EC 2.1.1.14). MetH catalyzes the final step in the remethylation of homocysteine which explains increased levels of homocysteine upon vitamin B12 deficiency. In animals, this may lead to blindness, neurological symptoms, and birth defects (Outteryck et al. 2012). MetH requires Cyanocobalamin reductase (CbIC) and Cobalamin trafficking protein (CbID) for proper function. $\mathrm{ClbC}$ catalyzes the decyanation of cyanocobalamin and the dealkylation of alkylcobalamins. In bacteria, an analog of CbIC/D, namely TonB, is involved in energy transduction for the uptake of cobalamin (Lerner-Ellis et al. 2006); (Hannibal et al. 2009). CbID interacts with CbIC and directs CbIC-cob(II)alamin molecules to the mitochondrion. Consistently, ClbC localizes either to cytoplasm or mitochondria, while ClbD remains in the cytosol (Gherasim et al. 2013; Mah et al. 2013). 
CblAdo transferase, cob(I)yrinic acid a,c-diamide adenosyltransferase, converts cobalamin into adenosylcobalamin (AdoCbl). AdoCbl is a cofactor of multiple enzymes that catalyze unusual rearrangement or elimination reactions. Some of them are restricted to prokaryotes e.g. lysine5,6-aminomutase, isobutyryl-CoA mutase and glutamate mutase. Others are present also in Eukaryotes eg. methylmalonyl-CoA mutase (Mera and Escalante-Semerena 2010); (Marsh and Meléndez 2012).

In humans, MM-CoA epimerase and MM-CoA mutase are both involved in fatty acid catabolism. MM-CoA epimerase catalyzes the rearrangement of (S)-methylmalonyl-CoA to the (R) form and uses a vitamin B12 cofactor (Overath et al. 1962). MM-CoA mutase induces the formation of adenosyl radical from AdoCbl cofactor and subsequently initiates a free-radical rearrangement of its substrate, (R)-methylmalonyl-CoA to succinyl-CoA - a key molecule of the citric acid cycle (Mancia et al. 1996). Methylmalonyl Co-A mutase-associated GTPase Cob (MeaB) is crucial for the proper functioning of methylmalonyl-CoA mutase (Takahashi-Iñiguez et al. 2017). Mutational analysis of this protein performed in Methylobacterium sp. showed an inability to convert methylmalonyl-CoA to succinyl-CoA caused by an inactive form of methylmalonyl-CoA mutase (Froese, Sean Froese, et al. 2010).

Kingdom Fungi comprises several lineages of non-Dikarya which, in the order of divergence, are classified into Chytrydiomycota (Chang et al. 2015; Liu and Stajich 2015) and Blastocladiomycota (Tabima et al. 2020) grouping many aquatic organisms, fully terrestrial animal-related Zoopagomycotina (Ahrendt et al. 2018), Entomophthoromycotina, Kickxellomycotina (Chang et al. 2015) and plant/soil/dung-associated Mucoromycotina (Mondo et al. 2017), Mortierellomycotina (Uehling et al. 2017) and Glomeromycotina (Chen et al. 2018). We can also list Microsporidia, that are described as the earliest diverging clade of fungi (Capella-Gutiérrez et al. 2012). The remaining Dikarya include evolutionary youngest and best-studied fungal phyla Ascomycota and Basidiomycota (Spatafora et al. 2016). None of the aforementioned B12-related enzymes has been reported from fungi. Yet non-Dikarya, early diverging lineages of fungi share multiple ancestral traits with animals and microbial eukaryotes. Here we show that all B12dependent eukaryotic pathways are present in non-Dikarya fungi as well. 


\section{Results}

Our initial searches showed that only eight enzymes are uniquely present in B12-dependent organisms (Table 1). All of them have their homologs within early-diverging fungal lineages (Supplementary Table S1). 
Table 1. B12-specific enzymes used for the identification of B12-dependent pathways in fungal proteomes with the total number of homologs identified in this study.

\begin{tabular}{|c|c|c|c|c|c|c|c|c|}
\hline $\begin{array}{l}\text { Human } \\
\text { gene }\end{array}$ & EC & Enzyme name & Reference & Pfam name & Pfam ID & $\begin{array}{l}\text { Structure } \\
\text { ID }\end{array}$ & $\begin{array}{l}\text { No. of } \\
\text { fungal } \\
\text { proteins }\end{array}$ & $\begin{array}{l}\text { No. of } \\
\text { fungal } \\
\text { species }\end{array}$ \\
\hline MUT & 5.4 .99 .2 & $\begin{array}{l}\text { Methylmalonyl-CoA mutase, } \\
\text { MM-CoA mutase }\end{array}$ & $\begin{array}{l}\text { (Forny et al. } \\
2014 \text { ) }\end{array}$ & MM_CoA_mutase & PF01642 & $2 \mathrm{XIJ}$ & 39 & 36 \\
\hline MCEE & 5.1 .99 .1 & $\begin{array}{l}\text { Methylmalonyl-CoA epimerase, } \\
\text { MM-CoA epimerase }\end{array}$ & $\begin{array}{l}\text { (Bobik and } \\
\text { Rasche 2001) }\end{array}$ & Glyoxalase_4 & PF13669 & $1 \mathrm{JC5}$ & 42 & 32 \\
\hline MMAA & 3.6.5.- & $\begin{array}{l}\text { Methylmalonyl Co-A mutase- } \\
\text { associated GTPase, MeaB }\end{array}$ & $\begin{array}{l}\text { (Bobik and } \\
\text { Rasche 2001; } \\
\text { Froese, } \\
\text { Kochan, et al. } \\
2010 \text { ) }\end{array}$ & MeaB & PF03308 & 2WWW & 30 & 24 \\
\hline MTR & 2.1.1.13 & Methionine synthase, MetH & $\begin{array}{l}\text { (Bobik and } \\
\text { Rasche 2001; } \\
\text { Froese, } \\
\text { Kochan, et al. } \\
\text { 2010; Bassila } \\
\text { et al. 2017) }\end{array}$ & Met_synt_B12 & PF02965 & $2 \mathrm{O} 2 \mathrm{~K}$ & 65 & 53 \\
\hline MMAB & 2.5.1.17 & $\begin{array}{l}\text { Methylmalonic aciduria and } \\
\text { homocystinuria type B family, } \\
\text { CblAdo transferase }\end{array}$ & $\begin{array}{l}\text { (Mera et al. } \\
2007 \text { ) }\end{array}$ & Cob_adeno_trans & PF01923 & $2 \mathrm{R} 6 \mathrm{X}$ & 71 & 50 \\
\hline MMACHC & 1.16.1.6 & $\begin{array}{l}\text { Methylmalonic aciduria and } \\
\text { homocystinuria type } C \text { family, } \\
\text { CbIC }\end{array}$ & $\begin{array}{l}\text { (Kim et al. } \\
\text { 2009) }\end{array}$ & $\mathrm{CblC}$ & PF16690 & $3 S B Z$ & 23 & 20 \\
\hline MMADHC & - & $\begin{array}{l}\text { Methylmalonic aciduria and } \\
\text { homocystinuria type D family, } \\
\text { CbID }\end{array}$ & $\begin{array}{l}\text { (Coelho et al. } \\
2008)\end{array}$ & CbID & PF10229 & $5 \mathrm{CV} 0$ & 55 & 47 \\
\hline- & 1.17.4.2 & $\begin{array}{l}\text { Ribonucleoside-diphosphate } \\
\text { reductase class II, RNR class II }\end{array}$ & $\begin{array}{l}\text { (Booker and } \\
\text { Stubbe 1993) }\end{array}$ & RNR_Alpha & PF17975 & 1L1L & 24 & 13 \\
\hline
\end{tabular}

\section{Distribution of B12 dependent enzymes in Fungi}

Cobalamin-dependent enzymes were identified in 50 out of 59 analyzed non-Dikarya fungi (Table 1, see Supplementary Table S2 for detailed lists of all protein accessions). This dataset contains all genome derived protein predictions for all non-Dikarya isolates deposited in GenBank by October 2019, with representatives of all main lineages.. The distribution of cobalamin-related enzymes among non-Dikarya fungi is shown in Fig. 1 and per organism occurrence of B12-related protein-coding genes is summarized in supplementary Table $\mathrm{S1}$ ). The whole set of studied enzymes is present in five non-Dikarya fungal proteomes, four of them belonging to the Glomeromycotina (Mucoromycota). The occurrence of cobalamin-related enzymes is common for all Mucoromycota species, but worth noting are the differences between Glomeromycotina, Mortierellomycotina, and Mucoromycotina (the latter comprising saprotrophic Mucorales, Umbelopsidales and plant symbionts Endogonales). In Mucorales only three families of 
cobalamin-dependent enzymes are conserved (CbID, MetH, and CblAdo transferase). For other Mortierallomycotina and Endogonales, it is common to retain four or more of the analyzed protein families. The whole set of enzymes can be found also in Blastocladiomycota. Other taxa with a high occurrence of cobalamin-dependent enzyme families are the animal-related Entomophthoromycotina, Kickxellomycotina, and Zoopagomycotina. All of them have homologs from four up to seven families.

Nine of the analyzed proteomes, all belonging to Chytridiomycota, do not contain any of the studied enzymes. The presence of six out of eight studied proteins in Chytridiales and Monoblepharidiales shows that not all Chytridiomycota are devoid of B12-dependent genes. Neocallimastigomycetes stand out especially here - none of the analyzed four proteomes from this taxon had any homologs of the cobalamin-related proteins family.

$x$ no homologs in taxon

homologs in $<50 \%$ of isolates in taxon

homologs in $\geq 50 \%$ of isolates in taxon
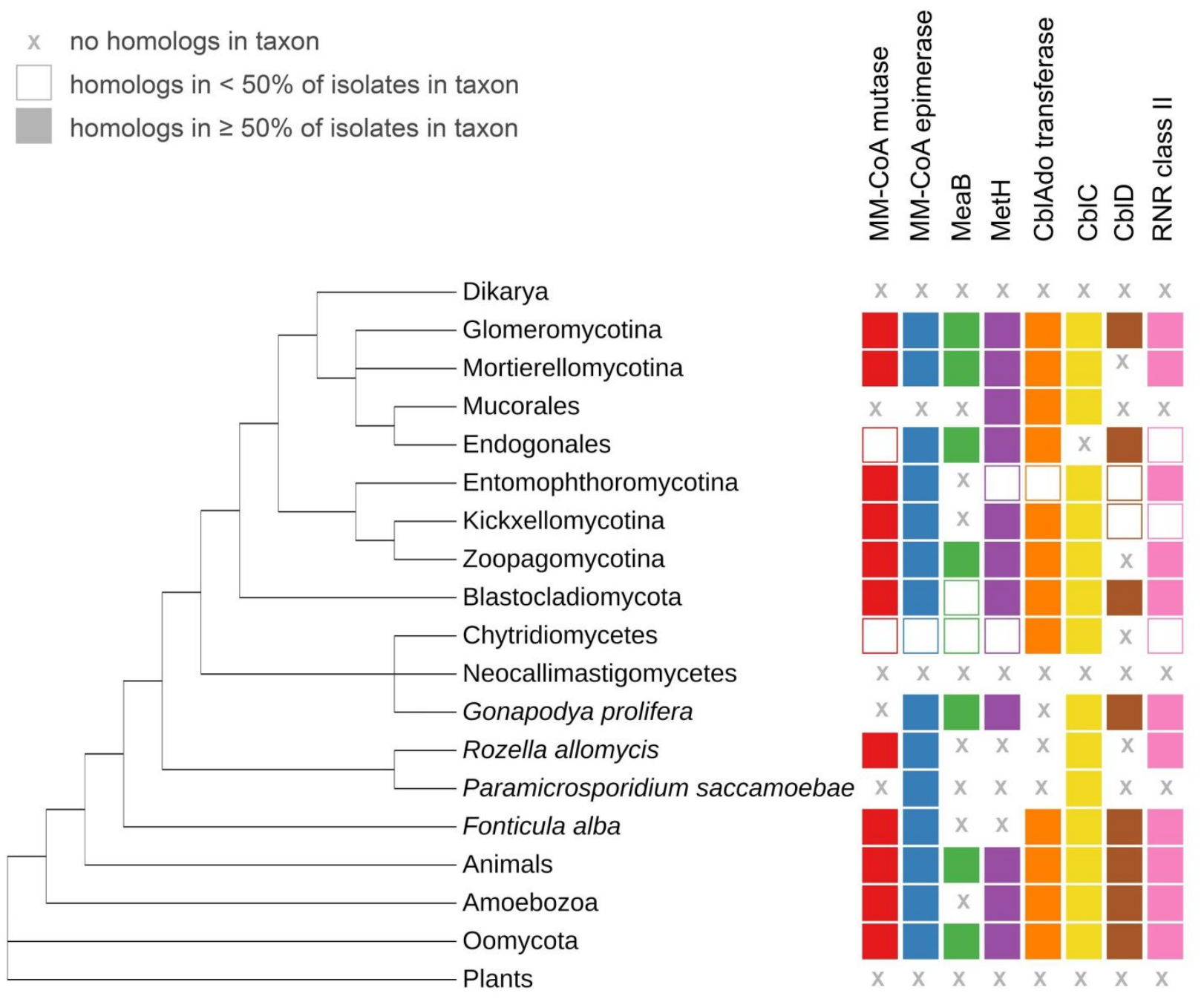
Figure 1. Distribution of B12-dependent protein families projected onto a partial eukaryotic tree of life. The schematic tree is based on (Davis et al. 2019; (Spatafora et al. 2016) for fungi and on (Burki et al. 2020) for remaining lineages.

\section{Conservation of B12 processing pathways}

Cobalamin-dependent enzymes play roles in three pathways associated with RNR class II, MetH, and MM-CoA mutase. Obtained results suggest that among early-diverging fungi there is a tendency to conserve the key enzymes rather than whole pathways. This is especially true in Mucorales which retained only CbIAdo transferase and part of MetH pathways. In other nonDikarya fungi, MM-CoA mutase associated pathway is also well conserved. The B12-dependent ribonucleotide reductase is least conserved but this might be associated with the presence of different RNR classes.

In order to ensure that all housekeeping functions provided by RNR class II, MM-CoA mutase, and MetH pathways are maintained in all of the studied isolates, even those devoid of B12dependent enzymes, we searched for cobalamin-independent alternatives. We looked for MetE which can substitute MetH (González et al. 1996), RNR class I instead of class II (Jordan and Reichard 1998), and methylcitrate cycle (MCC) as an alternative to MM-CoA mutase pathway (Dubey et al. 2013). We found that all these enzymes involved in B12-independent metabolic tracks can be identified in Dikarya and some of the non-Dikarya fungi providing the required enzymatic activity without B12 cofactor. The B12-independent alternatives are differently distributed among studied genomes compared to their B12-dependent counterparts. RNR class I and MCC synthase were found in all analyzed genomes and in other early-diverging fungi that have not been included in our analysis but were present in the NCBI NR database. Some earlydiverging fungi do not have B12-dependent enzymes at all for instance Batrachochytrium spp. have these two alternative B12-independent traits. Interestingly, both methionine synthases MetE and MetH can also co-occur in one genome - we observed co-occurrence of these two enzymes in 45 analysed genomes. MetE which is an alternative to B12-dependent MetH is present in 51 studied genomes, mostly in Mortierellomycotina but also in genomes that do not have B12dependent enzymes (e.g. Piromyces finnis). Seven Mortierellomycotina genomes, which were not included in our datasets, have also MetE copies. This might suggest that the metabolic pathways which first required B12 are still conserved among fungi, but have become independent of cobalamin for some organisms. 
Since most of the identified homologs of eight B12-dependent enzymes are annotated as hypothetical unknown proteins without experimental characterization, we performed tblastn searches on them against the NCBI EST database. This served as intermediate evidence that the predicted B12-related proteins in non-Dikarya fungi originate from active genes. Tblastn search results allow also to expect that genes encoding all identified proteins will be expressed.

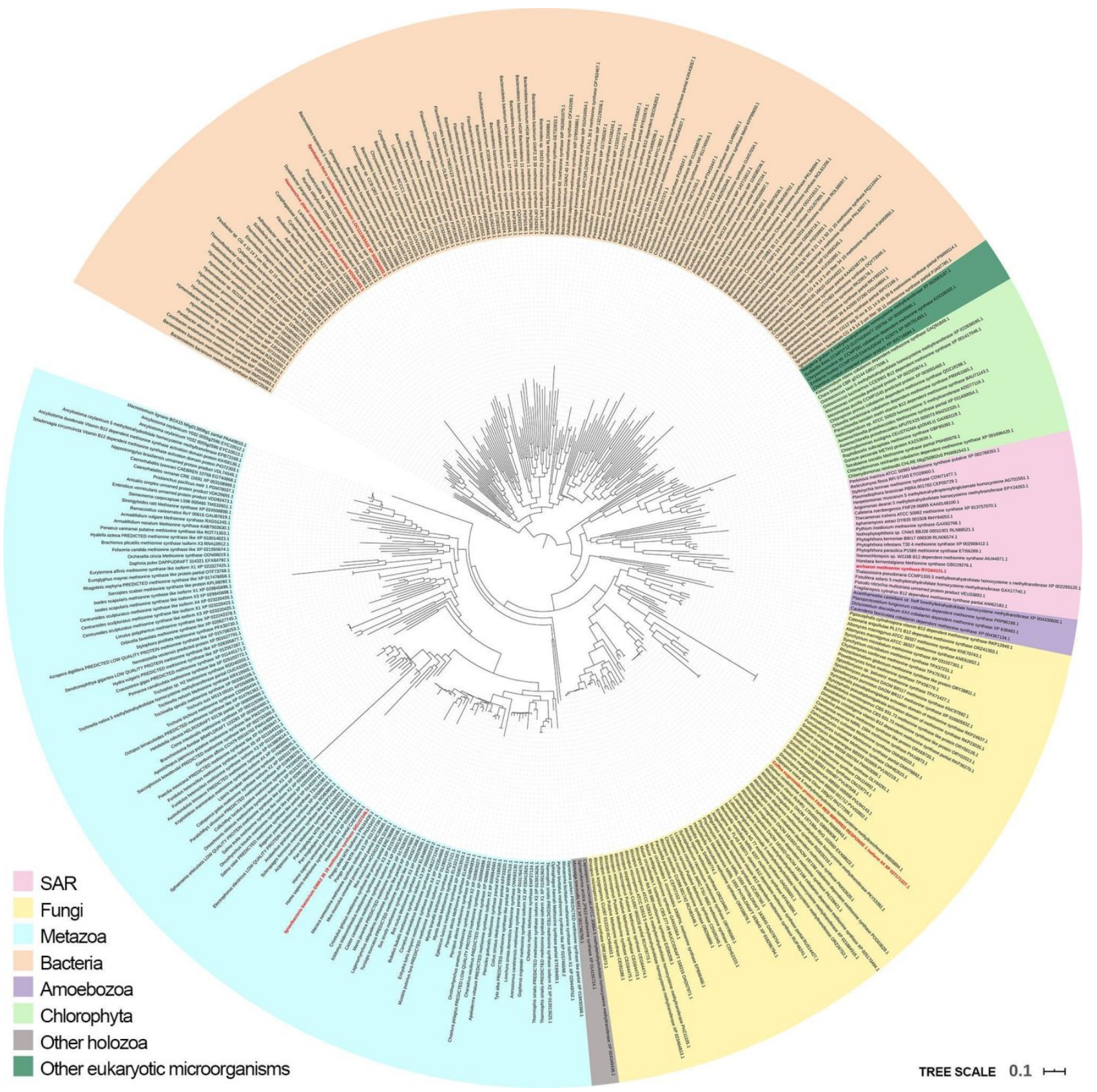

Figure 2. ML phylogenetic tree of methionine synthase MetH homologs. The tree was built based on 72 sequences from non-Dikaryal proteomes analysed in this study, aligned with their 
291 homologues from NCBI non-redundant database (Methods). Sequences marked with red labels are likely contaminations eg. Coffea in a fungal clade.

\section{Phylogenetic analysis of protein homologs associated with B12 utilization}

To trace the evolution of the studied proteins, phylogenetic trees for each of the eight protein families were inferred using Bayesian (BA) and Maximum Likelihood (ML) approaches (Supplementary Dataset DS1), except for MeaB and CbIAdo transferase (with highest numbers of identified homologs) where BA analyses did not converge to a reliable level of the standard deviation of split frequencies. We present an $\mathrm{ML}$ tree of MetH homologs as an example. All characteristic observations are common for all eight enzyme trees and are clearly visible on the MetH phylogenetic tree (Fig.2).

We noticed single bacterial sequences misannotated as fungal due to likely bacterial contamination of the fungal DNA samples. We also noticed single fungal sequences grouping within their bacterial relatives. In most cases these were proteins homologous to our enzyme yet with other function, e.g. MeaB is similar to other GTPases (KAA6408927.1). Non-Dikarya fungal sequences rarely grouped with bacterial sequences with the exception of MM-CoA mutase from Syncephalis pseudoplumigaleata (RKP28319.1, RKP28318.1) which displayed a very high sequence identity reaching $100 \%$ with Afipia alphaproteobacteria which might indicate sample contamination. Notably, misannotated Dikarya sequences, like other Dikarya representatives, could not be found in Eukaryotic clades for the analyzed enzymes.

Exclusively fungal clades can be observed in five protein families (Fig.3). For the other three enzymes, there are clades composed mostly of fungal homologs and ones belonging to other eukaryotic microorganisms (Holozoa, Amoebozoa, and SAR). Observed topologies in the eukaryotic part of the trees generally are congruent with the species tree. Interestingly, in two cases (CbIAdo transferase and RNR class II) Oomycota and Fungi clades are sisters to each other. Sequences identity of randomly chosen homologs is $\sim 63 \%$ for RNR class II (ETI40368.1 and KNE69215.1) and $~ 52 \%$ for CbIAdo transferase (XP_002997018.1 and KNE71581.1).

For MeaB, CbID, MetH, MM-CoA mutase, and RNR class II, fungal sequences form a sister clade to Fonticula alba (Holomycota), the closest relative of fungi belonging to Nucleariida. This pattern was observed for class II with the following score of aLTR support of the fungal clade: MeaB 1.00, CbID - 1.00, MetH - 1.00, MM-CoA mutase - 1.00, and RNR class II - 0.98. In the case of CbIAdo transferase, $\mathrm{CbIC}$ and $\mathrm{MM}-\mathrm{CoA}$ epimerase fungal sequences group together with either 
ancient Metazoa representatives (CblAdo transferase) or with microbial eukaryotes from Holomycota, Amoebozoa and SAR groups (MM-CoA epimerase). Importantly, non-Dikarya fungal sequences are always sister to other Eukaryotic sequences which rules out bacterial contamination. Sequences from model organisms belonging to diverse lineages of microbial eukaryotes, not only Opisthokonta, were represented in these clades, including representatives from Polysphondylium pallidum and Dictyostelium spp. (Amebozoa), Thecamonas trahens (Apusozoa), Chlamydomonas reinhardtii (Chlorophyta), Stentor coeruleus (Alveolata), Emiliania huxleyi (Haptophyta), Thalassiosira pseudonana, and Blastocystis spp. (Heterokonta), Naegleria gruberi and Euglena gracilis (Excavata). 

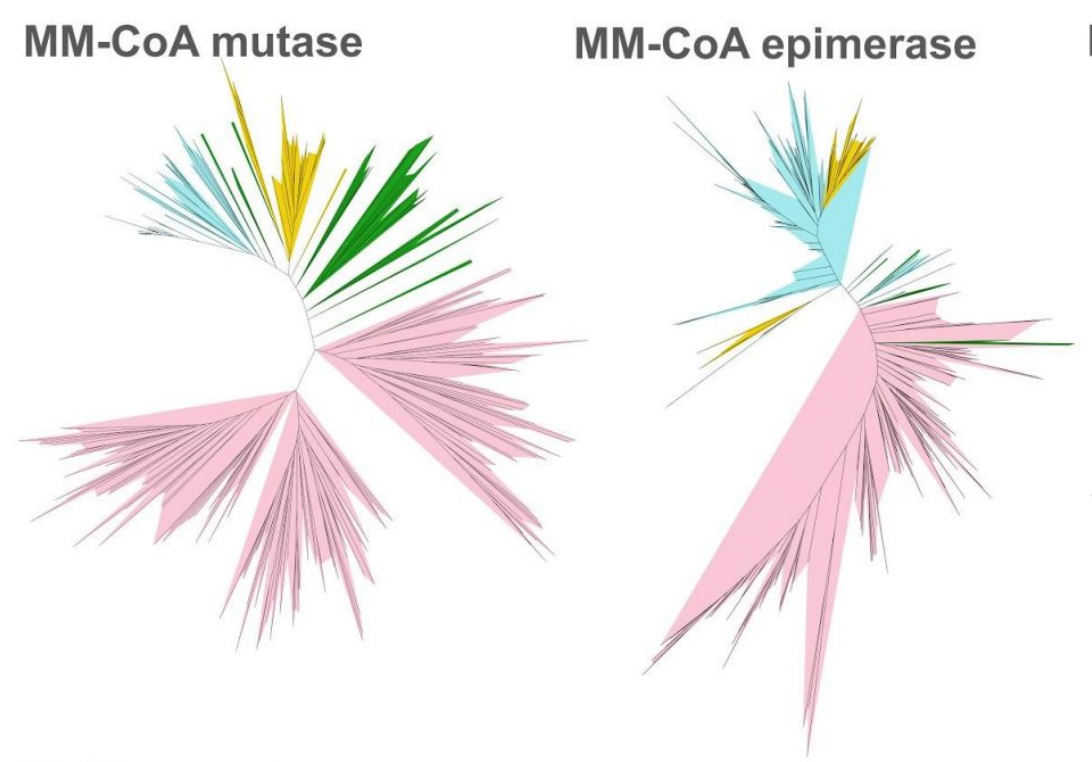

MeaB

MetH

CblAdo transferase
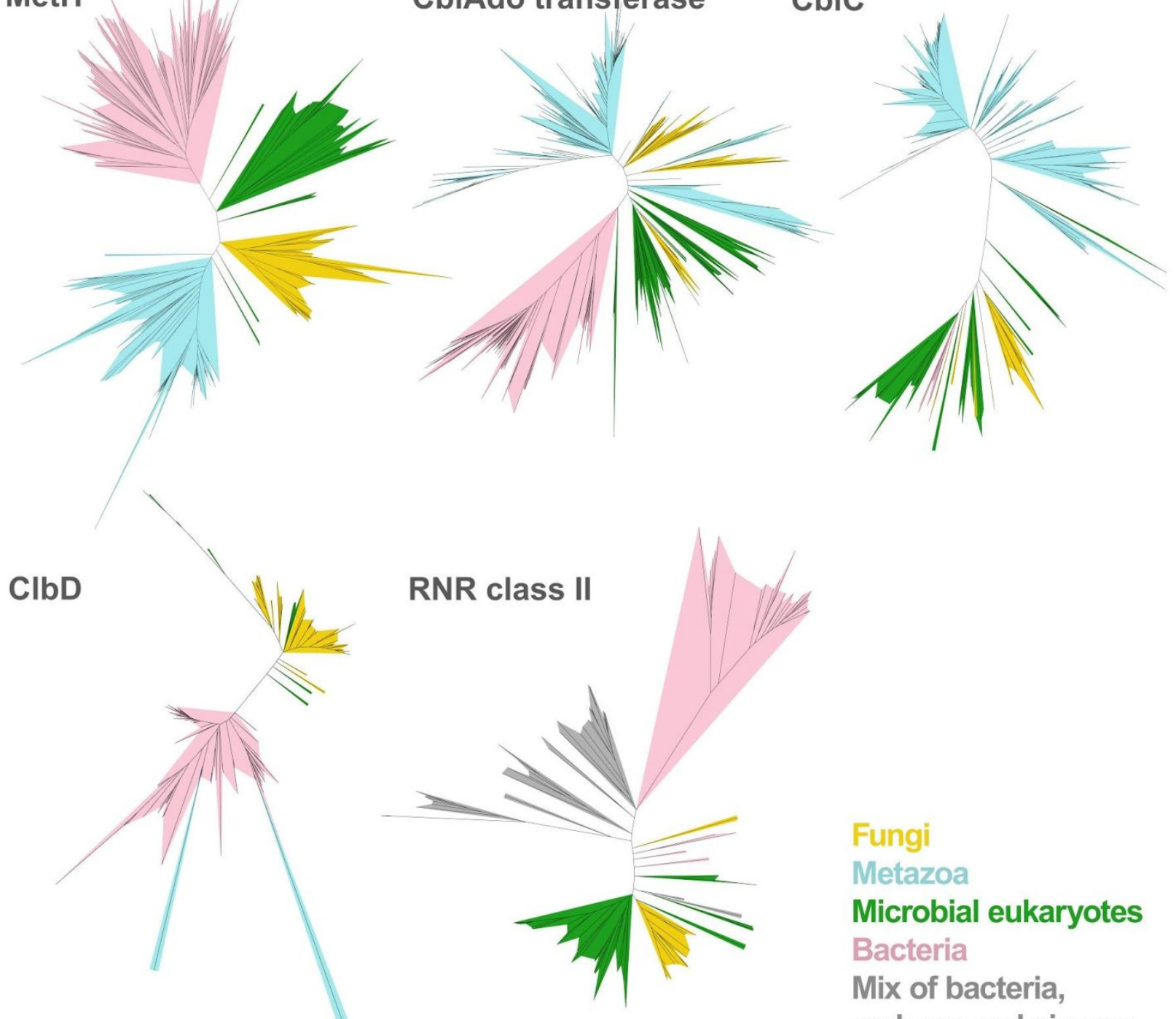

Fungi

Metazoa

Microbial eukaryotes

Bacteria

Mix of bacteria, archaea and viruses 
Figure 3. Unrooted ML trees of eight B12-related protein family representatives.

\section{Discussion}

Our discoveries contradict the current opinion that fungi neither synthesize nor use cobalamin (Jah et al. 2002; Duda, Pedziwilk, and Zodrow 1957) and do not have cobalt at all (Zhang, Ying, and $\mathrm{Xu}$ 2019). This claim remains true for Dikarya, but we demonstrate that the early diverging fungi do have proteins that either process or use cobalamin as a cofactor.

In Eukaryotes three main metabolic pathways use cobalamin - RNR class II, MM-CoA mutase and MetH pathways. Functions provided by these pathways are needed for the independent functioning of a living cell and can be lost in parasites (Zhang et al. 2009). Transport and trafficking of cobalamin in the cell is described in animals but homologs of the proteins responsible for the cobalamin transport e.g. LMBR1-like membrane protein transporters have a universal distribution in the Opisthokonta. This means that non-Dikarya fungi also have other components that are necessary for processing of cobalamin. Many of the enzymes involved in the MM-CoA mutase and MetH pathways, like mevalonate kinase and methionine synthase reductase, respectively, are conserved independently of B12 usage.

We found traces of all of these pathways among all lineages of early diverging fungi. The distribution of the genes encoding the above-mentioned enzymes is not uniform across the analyzed organisms. For non-Dikarya fungi, it is common to either have two out of three pathways or to have them incomplete.

Only Glomeromycotina and Blastocladiomycota have all three complete B12-dependent pathways. These two taxonomic groups are evolutionary and ecologically distant, they share only a few characteristics among others some of these fungi possess relatively big genomes. The latter may be a highlight of relaxed pressure on genome compactness.

The least conserved among fungal lineages is the RNR class II pathway. Organisms missing this class use cobalamin-independent RNR class I, which is the RNR used by animals. It is worth noticing that organisms are not limited to having only one class of RNRs at once (Jordan and Reichard 1998). Cobalamin-dependent RNR class II appears mostly in bacteria and, according to our results, also in non-Dikarya fungi and Oomycota. Additionally, RNR class II sequences from fungi and Oomycota form sister clades in phylogenetic trees (this is also true for CblAdo 
transferase homologs). This may suggest an ancient horizontal gene transfer between Oomycota and fungi resulting in nonidentical but highly similar sequences. This is yet another parallel molecular trait that groups fungi and other filamentous fungi-like organisms together, next to similarities in weaponry to attack plants (Latijnhouwers, de Wit, and Govers 2003), the evolution of the nitrate assimilation pathway (Ocaña-Pallarès et al. 2019), and the role of horizontal gene transfer (Soanes and Richards 2014; Rosewich and Kistler 2000). This trait is exquisitely interesting because it is shared by eukaryotic microorganisms but is absent from big multicellular forms.

The best-conserved pathway in non-Dikarya fungi - MetH - can be substituted with a cobalaminindependent enzyme called MetE (González et al. 1996). We checked if this enzyme variant also can occur in non-Dikarya fungi proteomes. MetE is present in all non-Dikarya fungi phyla, even in Neocallimastigomycetes, which do not have any other cobalamin-dependent or independent alternatives of studied pathways. For some of the non-Dikarya fungi, lack of CbIC protein can be observed. We did not look for substitutes for this protein, because the cooperation of CbIC and CbID in the MetH pathway was described only for animals - outside this group, the exact function of CbID protein is not documented, and perhaps in other organisms, CbIC is sufficient to perform its function by itself. One might speculate that other proteins are recruited to catalyze decyanation of cyanocobalamin and dealkylation of alkylcobalamins in non-animal organisms.

MM-CoA mutase pathway is more or less conserved among early-diverging fungal lineages. Interestingly, all Mucorales members lack all three B12-dependent enzymes of that pathway. We checked for alternatives for this metabolism track and it turned up to be more complex than in the other two cases. In Dikarya propionate metabolism is carried out in the methylcitrate cycle (MCC). Three key enzymes for this track are methylcitrate synthase (MCS), methylcitrate dehydrogenase (MCD), and methylisocitrate lyase (MCL) (Dubey et al. 2013). All of them are present in Dikarya and, interestingly also in Choanoflagellida and Metazoa, but not in early-diverging fungal and other ancient lineages like Ichthyosporea and in Fonticula. MCS and MCL are conserved as well in old fungal phyla as in Dikarya, but that does not apply to MCD. Following information about the MCC gene cluster (Santos et al. 2020) genomic context of this pathway was checked for Batrachochytrium and Mucor representatives showing no synteny. Moreover, no candidate dehydrogenases were found upstream or downstream of MCS and MCL genes. We assume that the function of MCD can be taken over by other dehydrogenases. 
According to our results, we can speculate the best-conserved elements of cobalamin-dependent pathways are key enzymes. For example, in the MetH pathway, the best-conserved element is MetH protein. On the contrary, it is quite common to lose CbIC and CbID proteins from the proteome (Supplementary Table S1). The question is why in some organisms only the main part of pathways is conserved and how it is possible for these pathways to work without helper protein. We speculate that our results may be biased towards the main enzymes because they are wellknown, especially have a well-known active site what allows for more rational data curation. Because our selection of potentially active homologs heavily relied on identified active site residues, it could have resulted in an underestimate of helper protein identification. Additionally, the MetH pathway is well described only in animal metabolism, so we cannot be sure about the role of CbIC and CbID in fungal metabolism and about the necessity of having these proteins. On the other hand, the best-conserved protein in the MM-CoA mutase pathway is CblAdo transferase. For Mucorales, it is common to have only this one protein from the whole MM-CoA mutase pathway. It is worth noticing that this protein is responsible for synthesizing AdoCbl cofactor for MM-CoA mutase which is the only protein in fungal metabolism that is known to require the AdoCbl cofactor. The question is why in Mucorales proteomes there is still pressure to conserve CbIAdo transferase while it is common to lose MM-CoA mutase.

Literature suggests that host-associated organisms have a tendency for the loss of cobalt utilization pathways (Zhang et al. 2009). Our results suggest that non-Dikarya fungi comply with this assumption. Chytridiomycota phylum combines amphibian parasites Batrachochytrium sp. and herbivorous mammals symbionts from class Neocallimastigomycetes. For these organisms, no cobalamin-dependent enzyme was found. These organisms may obtain the required resources from the host. However, our observations for plant-associated fungi are different. Mycorrhizal fungi from Glomeromycotina and Endogonales, despite maintaining extensive symbiotic relationships with $80 \%$ of plant species (Smith and Read 2010), retain well-conserved cobalamindependent pathways. It is possible that plant-associated non-Dikarya fungi kept these pathways simply because plant metabolism lacks cobalamin. The difference between plant and animal associated fungi may be a consequence of different pressures in such diverse ecological niches. Generally, parasites and obligate symbionts are biotrophs characterized by reduced genome size. However, in the case of mycorrhizal fungi, for a yet unknown reason, the pressure to reduce the genome seems to be relaxed (Lynch and Conery 2003); (James et al. 2020).

The question that still remains is what is the source of cobalamin for fungi. We speculate that fungi are able to accumulate B12 acquired from bacterial sources. B12 cofactor supply for at least 
some of the fungi with B12-dependent enzymes may be mediated by endohyphal bacteria with an intact B12 synthesis pathway. All crucial components of the B12 de novo synthesis pathway required for such a relationship were found in the case of symbiosis between Glomeromycotina fungus Gigaspora margarita and $\beta$-proteobacterium Candidatus Glomeribacter gigasporarum (Ghignone et al. 2012). Further experimental verification will be required to validate this hypothesis and confirm bacterial contribution to fungal B12 metabolism. There is also another open question of how fungi acquire the essential cofactor when growing in pure culture or in nature.

During the analysis of obtained results, we tried to understand the evolution of cobalamindependent metabolic pathways among kingdom Fungi. To widen the picture we checked studied proteomes for cobalamin-independent alternative metabolic pathways, and we confirmed their occurrence. Based on current knowledge we hypothesized that B12-dependent pathways are replaced by B12-independent alternatives in course of the evolution, and finally disappear in Dikarya lineages. In fact, the ability to utilize cobalamin is either retained or lost independently from the time of phyla divergence. Surprisingly we observed a correlation between the preservation of this ability and fungal ecology. As we observe, cobalamin-dependent pathways are more common in fungi associated with plants, than in species associated with animals and living as soil saprophytes. Correlation like this is unclear for mycoparasites. In our dataset, we have three fungi representing such a lifestyle and they have different enzyme distribution. In this case, we observed a correlation that mycoparasites which have B12-dependent enzymes infect fungi that also possess such enzymes (e.g. Rozella allomycis - Allomyces sp.). Similarly, parasites of organisms without B12-dependent genes do not have any of these enzymes themselves (Caulochytrium protostelioides - Sordaria sp.). This remark needs further investigation because we do not have enough data to form a reliable conclusion. Our discovery challenges the current view that fungi can neither synthesize nor utilize cobalamin. We proved that non-Dikarya fungal proteomes contain three metabolic pathways utilizing vitamin B12. We speculate these organisms have the possibility to accumulate cobalamin. Yet, these genomic observations need to be tested experimentally. Our discoveries may open the way for the selection of B12 over accumulating strains of food fermenting fungi without the need for genetic material manipulation. 


\section{B12 enzymes in other eukaryotes}

We also confirmed the occurrence of B12 related enzymes in other Holomycota taxa like Cryptomycota (Rozellida and Microsporidia) and Fonticulida. These organisms retain a maximum of only six out of the eight enzymes, but it is worth noting they are not independent, free-living organisms.

Studied enzymes are also present in Amoebozoa and Oomycota. Some other species from the SAR supergroup, to which Oomycota belongs (Burki et al. 2020), are known to have cobalamindependent methionine synthase (Boudouresque 2015). The matter is not clear about B12 utilization in Amoebozoa. There is contradictory information on the necessity to supplement the culture of Dictyostelium discoideum with that vitamin (Stephan et al. 2003). In addition, class II RNR has been observed in D. discoideum previously (Crona et al. 2013). B12-dependent enzymes are encountered also in green algae (Chlorophyta), red algae (Rhodophyta) (Croft et al. 2005; Thi Vu et al. 2013)) and Excavata (Helliwell et al. 2016). Green algae are known to acquire vitamin B12 through a symbiotic relationship with bacteria (Croft et al. 2005; Thi Vu et al. 2013).

Taken together, B12 dependence seems to be a widely distributed trait in Eukaryotes and was likely present in the last common ancestor of Eukaryotes. Several multicellular lineages including vascular plants and Dikarya developed B12-independent alternative pathways and, eventually, lost the B12 metabolism completely. The main question that remains about our discovery is the actual role of conserving B12-dependent enzymes in early diverging lineages of fungi - is this dependency on cobalamin operative or is it just a relic from shared ancestry with animals and amoebae?

\section{Methods}

Fifty-nine predicted non-Dikarya proteomes were downloaded from NCBI in October 2019 (Sayers et al. 2020) (Supplementary Table S3). Next, a pfam_scan.pl (default settings) (Mistry, Bateman, and Finn 2007) search of all protein sequences against a library of Pfam HMMs was performed. To expand our dataset NCBI NR database was searched for homologs of those nonDikarya fungal B12-dependent proteins and additionally for homologs of proteins from model eukaryotic organisms with known B12 dependent enzymes (Homo sapiens, Dictyostelium discoideum, Fonticula alba, Phytophthora infestans) using PSI-BLAST (evalue $=0.001$, num_iterations=3) (S. F. Altschul et al. 1997). The dataset was unified and clustered with CD-HIT 
( $n=4, c=0.7, a S=0.95, a L=0.95)$, all fungal hits were retained regardless of their sequence similarity. To get only homologs of a protein of our interest, there was a need to discard homologs from related protein families. To do this we visualized protein pairwise similarity using CLANS (Mistry, Bateman, and Finn 2007; Frickey and Lupas 2004) and selected separated groups of sequences. Dikarya sequences did not group together with the non-Dikarya-animal-protist clusters, except for single cases of clear contamination.

In the next step, sequences were aligned using local iterative mode in Mafft v. 3.7 (localpair, maxiterate=100) (Katoh et al. 2002). The alignment was additionally cleared manually from potential inactive homologs. All sequences that showed a lack of amino-acids crucial for enzyme activity or substitution of them with amino-acids that are not able to maintain enzyme activity, were discarded from the set.

All alignments were trimmed with TrimAl (model=gappyout) (Capella-Gutiérrez, Silla-Martínez, and Gabaldón 2009) to remove poorly conserved regions. Then, by using ProtTest (all-matrices, all-distributions) (Capella-Gutiérrez, Silla-Martínez, and Gabaldón 2009; Abascal, Zardoya, and Posada 2005), we appointed the best amino-acid substitution models based on Akaike Information Criterion AIC. Phylogenetic trees were built using LG model for each of the B12 metabolism-related enzymes with Bayesian (BA) and Maximum likelihood (ML) approaches using MrBayes 3.2.7a x86_64 (Huelsenbeck and Ronquist 2001) and PhyML (Guindon et al. 2010) respectively. $M L$ trees were estimated with a gamma distribution of rates between sites (four categories and alpha parameter estimated by PhyML) and aLRT Chi2-based parametric branch supports. In the course of BA inference, four Markov chains were run for 3 runs from random starting trees for $10^{7}$ generations, and trees were sampled every $2.5 \times 10^{2}$ generations. The first one-fourth of generations were discarded as burn-in. Then, we used the remaining samples to calculate the tree of maximum clade credibility.

Expression of representatives of each of the protein sets (three randomly chosen homologs from each family) was confirmed by tblastn (Stephen F. Altschul et al. 1990) (default settings) searches against the EST database at NCBI website.

Created phylogenetic trees were visualized and edited by iTOL v4 (Letunic and Bork 2019). Some bacterial sequences were misannotated as fungal due to likely bacterial contamination of the fungal DNA samples. A similar situation applies to some fungal sequences grouping within their bacterial relatives. In most cases, these proteins belong to another subfamily of homologous proteins with different substrate specificity. For instance, MeaB protein family groups diverse 
GTPases processing different substrates and only one of the subfamilies interacts with methylmalonyl-CoA mutase.

\section{Legends}

Table 1. B12-specific enzymes used for the identification of B12-dependent pathways in fungal proteomes with the total number of homologs identified in this study.

Figure 1. Distribution of B12-dependent protein families on a dendrogram showing a part of the eukaryotic tree of life, the schematic tree is based on (Davis et al. 2019, (Spatafora et al. 2016) for fungi and on (Burki et al. 2020) for remaining lineages. For each taxon, symbols on the right represent B12-dependent enzymes found in their proteome. $\mathrm{X}$ symbol means no identified homologs of the enzyme in the whole taxon; empty shape refers to the occurrence of the enzyme in less than half of studied representatives, filled shape means that half or more representatives have the enzyme in their proteomes.

Figure 2. Phylogenetic tree of methionine synthase MetH homologs. The tree was built based on 72 sequences from non-Dikaryal proteomes, aligned with their homologs from NCBI nonredundant database 291 (Methods). Sequences marked with red labels do not belong to organisms to which they were assigned.

Figure 3. Unrooted ML trees of eight B12-related protein family representatives.

\section{Acknowledgements}

We thank Anna Karnkowska, Julia Pawłowska, Krzysztof Pawłowski, and Marcin Grynberg for their insight and comments about the manuscript. This work was supported by the Polish National Science Centre (grant 2017/25/B/NZ2/01880 to AM).

\section{Data Availability Statement}

The analyses are based on publicly available sequences. All identifiers of analyzed proteins are listed in the Supplementary Materials. All trees built from those sequences are also listed in the Supplementary Materials. 


\section{Author Contributions}

A.M. designed the study, M.O. and A.M. prepared the data set and performed sequence analyses and M.O., K.S. and A.M. interpreted the data and wrote the manuscript.

\section{Competing interests}

The authors declare that they have no competing interests.

\section{Literature}

Abascal, Federico, Rafael Zardoya, and David Posada. 2005. "ProtTest: Selection of Best-Fit Models of Protein Evolution." Bioinformatics 21 (9): 2104-5.

Ahrendt, Steven R., C. Alisha Quandt, Doina Ciobanu, Alicia Clum, Asaf Salamov, Bill Andreopoulos, Jan-Fang Cheng, et al. 2018. "Leveraging Single-Cell Genomics to Expand the Fungal Tree of Life." Nature Microbiology 3 (12): 1417-28.

Altschul, S. F., T. L. Madden, A. A. Schäffer, J. Zhang, Z. Zhang, W. Miller, and D. J. Lipman. 1997. "Gapped BLAST and PSI-BLAST: A New Generation of Protein Database Search Programs." Nucleic Acids Research 25 (17): 3389-3402.

Altschul, Stephen F., Warren Gish, Webb Miller, Eugene W. Myers, and David J. Lipman. 1990. "Basic Local Alignment Search Tool." Journal of Molecular Biology. https://doi.org/10.1016/s0022-2836(05)80360-2.

Bassila, Christine, Rose Ghemrawi, Justine Flayac, D. Sean Froese, Matthias R. Baumgartner, Jean-Louis Guéant, and David Coelho. 2017. "Methionine Synthase and Methionine Synthase Reductase Interact with MMACHC and with MMADHC." Biochimica et Biophysica Acta, Molecular Basis of Disease 1863 (1): 103-12.

Bobik, T. A., and M. E. Rasche. 2001. "Identification of the Human Methylmalonyl-CoA Racemase Gene Based on the Analysis of Prokaryotic Gene Arrangements. Implications for Decoding the Human Genome." The Journal of Biological Chemistry 276 (40): 3719498.

Booker, S., and J. Stubbe. 1993. "Cloning, Sequencing, and Expression of the Adenosylcobalamin-Dependent Ribonucleotide Reductase from Lactobacillus Leichmannii." Proceedings of the National Academy of Sciences of the United States of America 90 (18): 8352-56.

Boudouresque, Charles-François. 2015. "Taxonomy and Phylogeny of Unicellular Eukaryotes." Environmental Microbiology: Fundamentals and Applications. https://doi.org/10.1007/97894-017-9118-2_7.

Burki, Fabien, Andrew J. Roger, Matthew W. Brown, and Alastair G. B. Simpson. 2020. "The New Tree of Eukaryotes." Trends in Ecology \& Evolution. https://doi.org/10.1016/j.tree.2019.08.008.

Capella-Gutiérrez, Salvador, José M. Silla-Martínez, and Toni Gabaldón. 2009. "trimAl: A Tool for Automated Alignment Trimming in Large-Scale Phylogenetic Analyses." Bioinformatics 25 (15): 1972-73.

Chang, Ying, Sishuo Wang, Satoshi Sekimoto, Andrea L. Aerts, Cindy Choi, Alicia Clum, Kurt M. LaButti, et al. 2015. "Phylogenomic Analyses Indicate That Early Fungi Evolved Digesting Cell Walls of Algal Ancestors of Land Plants." Genome Biology and Evolution 7 (6): 1590-1601. 
Chatthanawaree, W. 2011. "Biomarkers of Cobalamin (vitamin B12) Deficiency and Its Application." The Journal of Nutrition, Health \& Aging. https://doi.org/10.1007/s12603-0100280-x.

Chen, Eric C. H., Emmanuelle Morin, Denis Beaudet, Jessica Noel, Gokalp Yildirir, Steve Ndikumana, Philippe Charron, et al. 2018. "High Intraspecific Genome Diversity in the Model Arbuscular Mycorrhizal Symbiont Rhizophagus Irregularis." The New Phytologist 220 (4): 1161-71.

Coelho, David, Terttu Suormala, Martin Stucki, Jordan P. Lerner-Ellis, David S. Rosenblatt, Robert F. Newbold, Matthias R. Baumgartner, and Brian Fowler. 2008. "Gene Identification for the cbID Defect of Vitamin B12 Metabolism." The New England Journal of Medicine 358 (14): 1454-64.

Croft, Martin T., Andrew D. Lawrence, Evelyne Raux-Deery, Martin J. Warren, and Alison G. Smith. 2005. "Algae Acquire Vitamin B12 through a Symbiotic Relationship with Bacteria." Nature 438 (7064): 90-93.

Crona, Mikael, Lotta Avesson, Margareta Sahlin, Daniel Lundin, Andrea Hinas, Ralph Klose, Fredrik Söderbom, and Britt-Marie Sjöberg. 2013. "A Rare Combination of Ribonucleotide Reductases in the Social Amoeba Dictyostelium Discoideum." The Journal of Biological Chemistry 288 (12): 8198-8208.

Dereven'kov, I. A., D. S. Salnikov, R. Silaghi-Dumitrescu, S. V. Makarov, and O. I. Koifman. 2016. "Redox Chemistry of Cobalamin and Its Derivatives." Coordination Chemistry Reviews. https://doi.org/10.1016/j.ccr.2015.11.001.

Dubey, Mukesh K., Anders Broberg, Dan Funck Jensen, and Magnus Karlsson. 2013. "Role of the Methylcitrate Cycle in Growth, Antagonism and Induction of Systemic Defence Responses in the Fungal Biocontrol Agent Trichoderma Atroviride." Microbiology 159 (Pt 12): 2492-2500.

Duda, J., Z. Pedziwilk, and K. Zodrow. 1957. "[Studies on the appearance of vitamin B12 in leguminous plants]." Acta microbiologica Polonica 6 (3): 233-38.

Forny, Patrick, D. Sean Froese, Terttu Suormala, Wyatt W. Yue, and Matthias R. Baumgartner. 2014. "Functional Characterization and Categorization of Missense Mutations That Cause Methylmalonyl-CoA Mutase (MUT) Deficiency." Human Mutation 35 (12): 1449-58.

Frank, S., A. A. Brindley, E. Deery, P. Heathcote, A. D. Lawrence, H. K. Leech, R. W. Pickersgill, and M. J. Warren. 2005. "Anaerobic Synthesis of Vitamin B12: Characterization of the Early Steps in the Pathway." Biochemical Society Transactions 33 (Pt 4): 811-14.

Frickey, T., and A. Lupas. 2004. "CLANS: A Java Application for Visualizing Protein Families Based on Pairwise Similarity." Bioinformatics. https://doi.org/10.1093/bioinformatics/bth444.

Froese, D. Sean, Grazyna Kochan, João R. C. Muniz, Xuchu Wu, Carina Gileadi, Emelie Ugochukwu, Ewelina Krysztofinska, Roy A. Gravel, Udo Oppermann, and Wyatt W. Yue. 2010. "Structures of the Human GTPase MMAA and Vitamin B12-Dependent Methylmalonyl-CoA Mutase and Insight into Their Complex Formation." The Journal of Biological Chemistry 285 (49): 38204-13.

Froese, D. Sean, D. Sean Froese, Grazyna Kochan, João R. C. Muniz, Xuchu Wu, Carina Gileadi, Emelie Ugochukwu, et al. 2010. "Structures of the Human GTPase MMAA and Vitamin B12-Dependent Methylmalonyl-CoA Mutase and Insight into Their Complex Formation." Journal of Biological Chemistry. https://doi.org/10.1074/jbc.m110.177717.

Gherasim, Carmen, Luciana Hannibal, Deepa Rajagopalan, Donald W. Jacobsen, and Ruma Banerjee. 2013. "The C-Terminal Domain of CbID Interacts with CbIC and Influences Intracellular Cobalamin Partitioning." Biochimie 95 (5): 1023-32.

Ghignone, Stefano, Alessandra Salvioli, Iulia Anca, Erica Lumini, Giuseppe Ortu, Luca Petiti, Stéphane Cruveiller, et al. 2012. "The Genome of the Obligate Endobacterium of an AM Fungus Reveals an Interphylum Network of Nutritional Interactions." The ISME Journal 6 (1): 136-45. 
González, J. C., K. Peariso, J. E. Penner-Hahn, and R. G. Matthews. 1996. "CobalaminIndependent Methionine Synthase from Escherichia Coli: A Zinc Metalloenzyme."

Biochemistry 35 (38): 12228-34.

Grenville-Briggs, Laura J., Anna O. Avrova, Catherine R. Bruce, Alison Williams, Stephen C. Whisson, Paul R. J. Birch, and Pieter van West. 2005. "Elevated Amino Acid Biosynthesis in Phytophthora Infestans during Appressorium Formation and Potato Infection." Fungal Genetics and Biology: FG \& B 42 (3): 244-56.

Guindon, Stéphane, Jean-François Dufayard, Vincent Lefort, Maria Anisimova, Wim Hordijk, and Olivier Gascuel. 2010. "New Algorithms and Methods to Estimate Maximum-Likelihood Phylogenies: Assessing the Performance of PhyML 3.0." Systematic Biology 59 (3): 30721.

Hannibal, Luciana, Jihoe Kim, Nicola E. Brasch, Sihe Wang, David S. Rosenblatt, Ruma Banerjee, and Donald W. Jacobsen. 2009. "Processing of Alkylcobalamins in Mammalian Cells: A Role for the MMACHC (cblC) Gene Product." Molecular Genetics and Metabolism 97 (4): 260-66.

Helliwell, Katherine Emma, Andrew David Lawrence, Andre Holzer, Ulrich Johan Kudahl, Severin Sasso, Bernhard Kräutler, David John Scanlan, Martin James Warren, and Alison Gail Smith. 2016. "Cyanobacteria and Eukaryotic Algae Use Different Chemical Variants of Vitamin B12." Current Biology: CB 26 (8): 999-1008.

Herrick, John, and Bianca Sclavi. 2007. "Ribonucleotide Reductase and the Regulation of DNA Replication: An Old Story and an Ancient Heritage." Molecular Microbiology 63 (1): 22-34. Huelsenbeck, J. P., and F. Ronquist. 2001. "MRBAYES: Bayesian Inference of Phylogenetic Trees." Bioinformatics. https://doi.org/10.1093/bioinformatics/17.8.754.

Jah, J-H Martens H. Barg M. Warren D., J-H Martens, H. Barg, M. Warren, and D. Jah. 2002. "Microbial Production of Vitamin B 12." Applied Microbiology and Biotechnology. https://doi.org/10.1007/s00253-001-0902-7.

James, Timothy Y., Jason E. Stajich, Chris Todd Hittinger, and Antonis Rokas. 2020. "Toward a Fully Resolved Fungal Tree of Life." Annual Review of Microbiology 74 (September): 291313.

Jordan, A., and P. Reichard. 1998. "Ribonucleotide Reductases." Annual Review of Biochemistry 67: 71-98.

Katoh, Kazutaka, Kazuharu Misawa, Kei-Ichi Kuma, and Takashi Miyata. 2002. "MAFFT: A Novel Method for Rapid Multiple Sequence Alignment Based on Fast Fourier Transform." Nucleic Acids Research 30 (14): 3059-66.

Kim, Jihoe, Luciana Hannibal, Carmen Gherasim, Donald W. Jacobsen, and Ruma Banerjee. 2009. "A Human Vitamin B12 Trafficking Protein Uses Glutathione Transferase Activity for Processing Alkylcobalamins." The Journal of Biological Chemistry 284 (48): 33418-24.

Kolberg, M. 2004. "Structure, Function, and Mechanism of Ribonucleotide Reductases." Biochimica et Biophysica Acta (BBA) - Proteins \& Proteomics. https://doi.org/10.1016/s1570-9639(04)00054-8.

Kräutler, Bernhard. 2012. "Biochemistry of B12-Cofactors in Human Metabolism." Sub-Cellular Biochemistry 56: 323-46.

Larsson, Karl-Magnus, Albert Jordan, Rolf Eliasson, Peter Reichard, Derek T. Logan, and Pär Nordlund. 2004. "Structural Mechanism of Allosteric Substrate Specificity Regulation in a Ribonucleotide Reductase." Nature Structural \& Molecular Biology 11 (11): 1142-49. Latijnhouwers, Maita, Pierre J. G. M. de Wit, and Francine Govers. 2003. "Oomycetes and Fungi: Similar Weaponry to Attack Plants." Trends in Microbiology 11 (10): 462-69. Lerner-Ellis, Jordan P., Jamie C. Tirone, Peter D. Pawelek, Carole Doré, Janet L. Atkinson, David Watkins, Chantal F. Morel, et al. 2006. "Identification of the Gene Responsible for Methylmalonic Aciduria and Homocystinuria, cblC Type." Nature Genetics 38 (1): 93-100. Letunic, Ivica, and Peer Bork. 2019. "Interactive Tree Of Life (iTOL) v4: Recent Updates and 
New Developments." Nucleic Acids Research 47 (W1): W256-59.

Liu, Peng, and Jason E. Stajich. 2015. "Characterization of the Carbohydrate Binding Module 18 Gene Family in the Amphibian Pathogen Batrachochytrium Dendrobatidis." Fungal Genetics and Biology: FG \& B 77 (April): 31-39.

Lynch, Michael, and John S. Conery. 2003. "The Origins of Genome Complexity." Science 302 (5649): 1401-4.

Mah, Wayne, Justin C. Deme, David Watkins, Stephen Fung, Alexandre Janer, Eric A. Shoubridge, David S. Rosenblatt, and James W. Coulton. 2013. "Subcellular Location of MMACHC and MMADHC, Two Human Proteins Central to Intracellular Vitamin B(12) Metabolism." Molecular Genetics and Metabolism 108 (2): 112-18.

Mancia, Filippo, Nicholas H. Keep, Atsushi Nakagawa, Peter F. Leadlay, Sean McSweeney, Bjarne Rasmussen, Peter Bö Secke, Olivier Diat, and Philip R. Evans. 1996. "How Coenzyme B12 Radicals Are Generated: The Crystal Structure of MethylmalonylCoenzyme A Mutase at 2 å Resolution." Structure. https://doi.org/10.1016/s09692126(96)00037-8.

Marsh, E. Neil G., and Gabriel D. Román Meléndez. 2012. "Adenosylcobalamin Enzymes: Theory and Experiment Begin to Converge." Biochimica et Biophysica Acta 1824 (11): 1154-64.

Mera, Paola E., and Jorge C. Escalante-Semerena. 2010. "Multiple Roles of ATP:cob(I)alamin Adenosyltransferases in the Conversion of B12 to Coenzyme B12." Applied Microbiology and Biotechnology 88 (1): 41-48.

Mera, Paola E., Martin St Maurice, Ivan Rayment, and Jorge C. Escalante-Semerena. 2007. "Structural and Functional Analyses of the Human-Type Corrinoid Adenosyltransferase (PduO) from Lactobacillus Reuteri." Biochemistry 46 (48): 13829-36.

Mistry, Jaina, Alex Bateman, and Robert D. Finn. 2007. "Predicting Active Site Residue Annotations in the Pfam Database." BMC Bioinformatics 8 (August): 298.

Mondo, Stephen J., Richard O. Dannebaum, Rita C. Kuo, Katherine B. Louie, Adam J. Bewick, Kurt LaButti, Sajeet Haridas, et al. 2017. "Widespread Adenine N6-Methylation of Active Genes in Fungi." Nature Genetics 49 (6): 964-68.

Ocaña-Pallarès, Eduard, Sebastián R. Najle, Claudio Scazzocchio, and Iñaki Ruiz-Trillo. 2019. "Reticulate Evolution in Eukaryotes: Origin and Evolution of the Nitrate Assimilation Pathway." PLoS Genetics 15 (2): e1007986.

Outteryck, Olivier, Jérôme de Sèze, Tanya Stojkovic, Jean-Marie Cuisset, Dries Dobbelaere, Sophie Delalande, Arnaud Lacour, et al. 2012. "Methionine Synthase Deficiency: A Rare Cause of Adult-Onset Leukoencephalopathy." Neurology 79 (4): 386-88.

Overath, P., G. M. Kellerman, F. Lynen, H. P. Fritz, and H. J. Keller. 1962. "[On the mechanism of the rearrangement of methylmalonyl-Co A into succinyl-Co A. II. Experiments on the mechanism of action of methylmalonyl-Co A isomerase and methylmalonyl-Co A racemase]." Biochemische Zeitschrift 335: 500-518.

Rosewich, U. Liane, and H. Corby Kistler. 2000. "Role of Horizontal Gene Transfer in the Evolution of Fungi." Annual Review of Phytopathology 38 (September): 325-63.

Roth, J. R., J. G. Lawrence, M. Rubenfield, S. Kieffer-Higgins, and G. M. Church. 1993. "Characterization of the Cobalamin (vitamin B12) Biosynthetic Genes of Salmonella Typhimurium." Journal of Bacteriology 175 (11): 3303-16.

Santos, Luiz Paulo Araújo, Leandro do Prado Assunção, Patrícia de Souza Lima, Gabriel Brum Tristão, Matthias Brock, Clayton Luiz Borges, Mirelle Garcia Silva-Bailão, Célia Maria de Almeida Soares, and Alexandre Melo Bailão. 2020. "Propionate Metabolism in a Human Pathogenic Fungus: Proteomic and Biochemical Analyses." IMA Fungus. https://doi.org/10.1186/s43008-020-00029-9.

Sayers, Eric W., Jeff Beck, J. Rodney Brister, Evan E. Bolton, Kathi Canese, Donald C. Comeau, Kathryn Funk, et al. 2020. "Database Resources of the National Center for 
Biotechnology Information." Nucleic Acids Research 48 (D1): D9-16.

Smith, Sally E., and David J. Read. 2010. Mycorrhizal Symbiosis. Academic Press.

Soanes, Darren, and Thomas A. Richards. 2014. "Horizontal Gene Transfer in Eukaryotic Plant Pathogens." Annual Review of Phytopathology 52: 583-614.

Spatafora, Joseph W., Ying Chang, Gerald L. Benny, Katy Lazarus, Matthew E. Smith, Mary L. Berbee, Gregory Bonito, et al. 2016. "A Phylum-Level Phylogenetic Classification of Zygomycete Fungi Based on Genome-Scale Data." Mycologia 108 (5): 1028-46.

Stephan, Miriam, Usama Beshay, Karl Friehs, and Erwin Flaschel. 2003. "Influence of Medium Composition on Growth Behaviour of Dictyostelium Discoideum for Cultivation on Axenic Media." Process Biochemistry 39 (3): 333-43.

Tabima, Javier F., Ian A. Trautman, Ying Chang, Yan Wang, Stephen Mondo, Alan Kuo, Asaf Salamov, Igor V. Grigoriev, Jason E. Stajich, and Joseph W. Spatafora. 2020.

"Phylogenomic Analyses of Non-Dikarya Fungi Supports Horizontal Gene Transfer Driving Diversification of Secondary Metabolism in the Amphibian Gastrointestinal Symbiont,." G3 10 (9): 3417-33.

Takahashi-Iñiguez, Toshiko, Alfonso González-Noriega, Colette Michalak, and María Elena Flores. 2017. "Human MMAA Induces the Release of Inactive Cofactor and Restores Methylmalonyl-CoA Mutase Activity through Their Complex Formation." Biochimie 142 (November): 191-96.

Thi Vu, Hoan, Hideomi Itoh, Satoshi Ishii, Keishi Senoo, and Shigeto Otsuka. 2013. "Identification and Phylogenetic Characterization of Cobalamin Biosynthetic Genes of Ensifer Adhaerens." Microbes and Environments / JSME 28 (1): 153-55.

Uehling, J., A. Gryganskyi, K. Hameed, T. Tschaplinski, P. K. Misztal, S. Wu, A. Desirò, et al. 2017. "Comparative Genomics of Mortierella Elongata and Its Bacterial Endosymbiont Mycoavidus Cysteinexigens." Environmental Microbiology 19 (8): 2964-83.

Zhang, Yan, Dmitry A. Rodionov, Mikhail S. Gelfand, and Vadim N. Gladyshev. 2009. "Comparative Genomic Analyses of Nickel, Cobalt and Vitamin B12 Utilization." BMC Genomics 10 (February): 78.

Zhang, Yan, Huimin Ying, and Yinzhen Xu. 2019. "Comparative Genomics and Metagenomics of the Metallomes." Metallomics. https://doi.org/10.1039/c9mt00023b. 
$x$ no homologs in taxon

homologs in $<50 \%$ of isolates in taxon

homologs in $\geq 50 \%$ of isolates in taxon
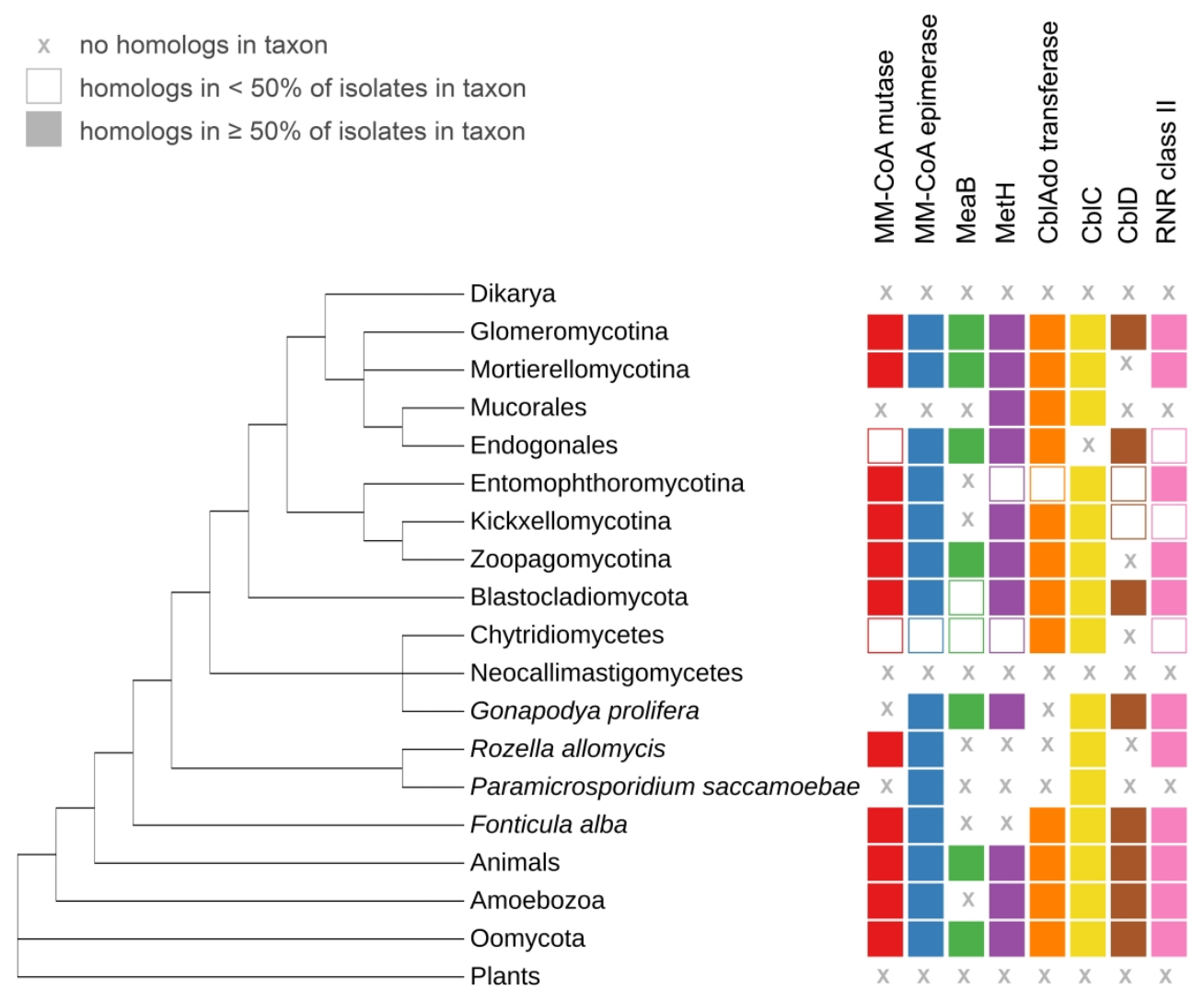

Figure 1. Distribution of B12-dependent protein families projected onto a partial eukaryotic tree of life. The schematic tree is based on (Davis et al. 2019; (Spatafora et al. 2016) for fungi and on (Burki et al. 2020) for remaining lineages. 


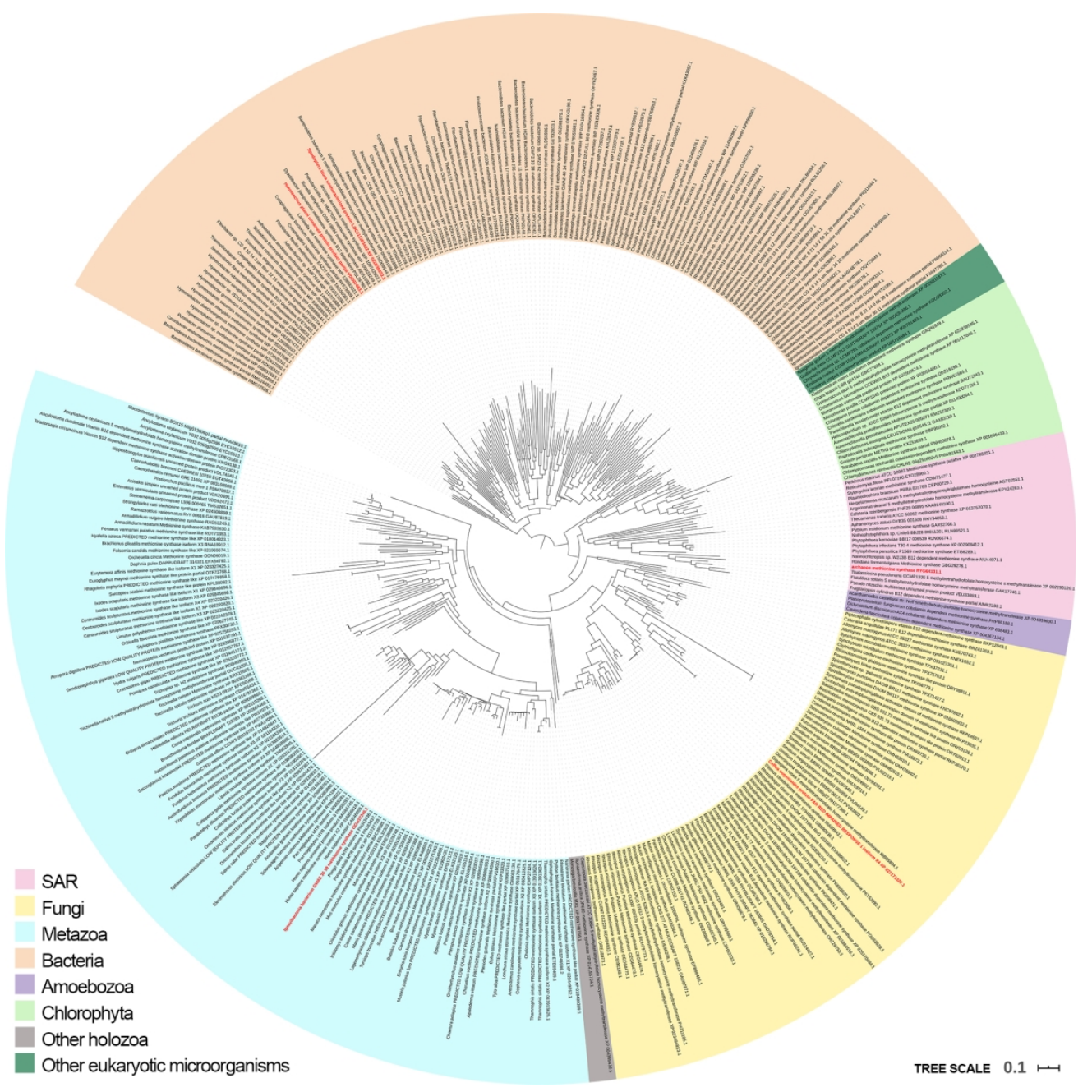

Figure 2. ML phylogenetic tree of methionine synthase MetH homologs. The tree was built based on 72 sequences from non-Dikaryal proteomes analysed in this study, aligned with their 291 homologues from NCBI non-redundant database (Methods). Sequences marked with red labels are likely contaminations eg. Coffea in a fungal clade. 


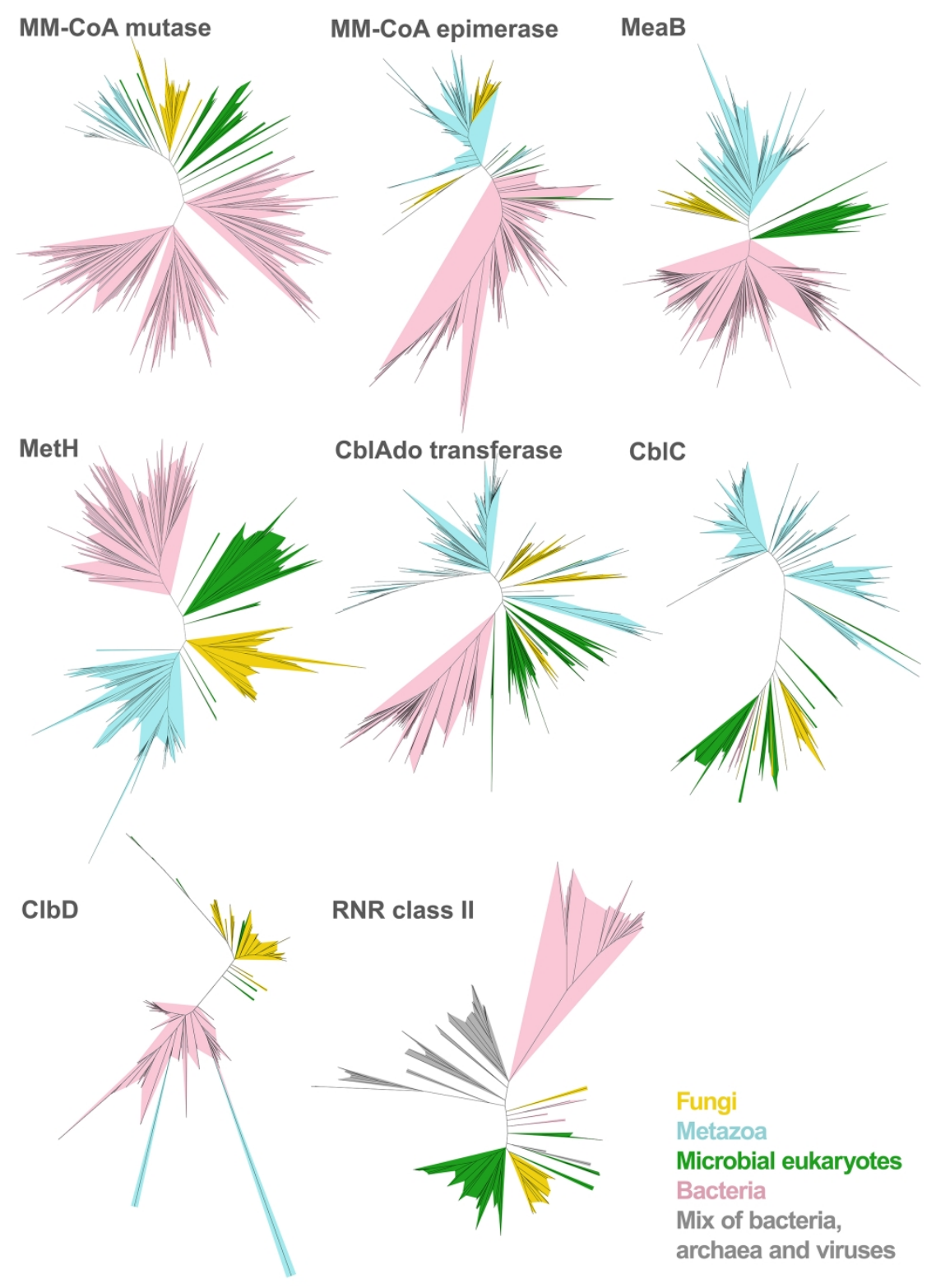

Figure 3. Unrooted ML trees of eight B12-related protein family representatives. 